\title{
XXI.
}

\section{Eine scheinbare bedeutende Drehung von Objecten bei Neigung des Kopfes nach rechts oder links.}

\author{
Von Hermann Aubert, \\ privatdocent all der Universität zu Lreslau.
}

Bei Untersuchungen, die ich in einem verfinsterten Zimmer behufs verschiedener physiologisch-optischer Probleme anstellte, fiel mir die Drehung eines quadratischen Ausschnittes auf, welcher dazu diente, verschiedene bestimmbare Mengen von Licht in das Zimmer einzulassen. Die quadratische Oeffnung stand mit ibren Seiten genau senkrecht und wagerecht; als ich aber, neben derselben stehend, den Kopf so neigte, dass die Grundlinie (die Verbindungslinie der beiden Augenmittelpunkte) vertikal stand, so erschien mir die Oeffnung wie ein Karten-Carreau. Dass eine Verschiebung des Apparates, wie ich zuerst vermuthete, nicht daran schuld war, davon überzeugte ich mich bald; denn als ich bei gewöhnlicher Kopfstellung die Oeffnung betrachtete, hatte sie ihre ursprüngliche lage. Ging ich nun allmälig aus der Normalstellung des Kopfes zu der Neigung des Kopfes nach rechts und links über, so fand dabei jedesmal eine Drehung des Quadrates statt, die mit der Neigung des Kopfes zu- und abnahm.

Eine weitere Berücksichtigung schien mir diese Beobachtung um so mehr zu verdienen, als Donders in seinem berühmten „Beitrag zur Lehre von den Bewegungen des menschlichen Auges" (Holländische Beiträge, p. 105) die Neigungen des Kopfes nach der rechten und linken Seite nicht in den Kreis seiner Untersuchungen gezogen hat, und auch nach ihm keine Untersuchungen darüber angestellt worden zu sein scheinen.

Aus verschiedenen Gründen wendete ich statt der quadratischen Oeffnung einen linearen Ausschnitt von $5 \mathrm{Ctm}$. Länge und 
$2 \mathrm{Ctm}$. Breite an, den ich im Folgenden als ", belle Linie" bezeichnen werde und beobachtete nun Folgendes:

War die helle Linie vertikal, so erschien sie, wenn ich den Kopf nach rechts neigte, so dass also das rechte $0 \mathrm{hr}$ nach unten gerichtet war, schief, und zwar von rechts unten nach links oben gerichtet; neigte ich den Kopf nach links, so erschien die Linie von links unten nach rechts oben gerichtet. Entsprechend waren die Resultate bei horizontaler Lage der hellen Linie: sie erschien bei Neigung des Kopfes nach der rechten Seite von links unten nach rechts oben, bei nach links geneigtem Kopfe dagegen von rechts unten nach links oben gerichtet.

Um über die Grösse der scheinbaren Drehung einen Anhalt zu gewinnen, gab ich der Linie eine wirkliche Lage von $45^{\circ}$ und zwar war sie von links unten nach rechts oben gerichtet. Beugte ich nun den Kopf nach rechts, so wurde sie vertikal, ja sie ging bei sehr starker Neigung des Kopfes über die vertikale Stellung hinaus und erschien von rechts unten nach links oben geneigt. Wie zu erwarten, wurde die Linie, wenn ich den Kopf nach links neigte, horizontal, ging aber bei weiterer Neigung über die horizontale hinaus, so dass sie wieder ein wenig von rechts unten nach links oben geneigt schien. Dasselbe zeigte sich mutatis mutandis bei einer Neigung der Linie von rechts unten nach links oben. Daraus geht hervor, dass die scheinbare Drehung der hellen Linie für die beiderseitigen Neigungen des Kopfes über $90^{\circ}$ beträgt, und dass zweitens bei einer Neigung des Kopfes um $90^{\circ}$ und mehr die scheinbare Drehung der bellen Linje nur $45^{\circ}$ oder etwas darüber oder darunter ausmacht.

Das Maximum der scheinbaren Drehung tritt ein, wenn man den Kopf um etwa $135^{\circ}$ geneigt hat; geht man darüber hinaus, so geht die Linie in ihre wirkliche Lage zurück. Sieht man zwischen den Beinen durch oder steht man auf dem Kopfe, so erscheint die helle linie ebenso wie bei gewöhnlicher aufrechter Kopfstellung.

Die Drehung der hellen Linie folgt der Neigung des Kopfes, wenn diese langsam ausgeführt wird, ziemlich unmittelbar; neigt man aber den Kopf plötzlich bedeutend, so vergehen einige Se- 
kunden, bevor die Linie ihre Drehung vollendet. Auch bei sehr geringer Neigung des Kopfes dreht sich die Linie scheinbar, wenn auch nur wenig.

Die, Drehung der hellen Linie erfolgt umgekehrt wie die Drehung des vertikalen Meridians oder der Grundlinie: wenn ich den Kopf nach links neige, so drebt sich die Linie in dem Sinne eines Uhrzeigers.

Die Erscheinung findet nur statt, wenn das Zimmer so stark verdunkelt ist, dass keine Gegenstände sichtbar sind, nach denen man sich orientiren kann. Sobald dergleichen sichtbar werden, so erscheint die Linie in ihrer wirklichen Lage. Hat man z. B. der Linie eine schiefe Lage unter einem Winkel von $45^{\circ}$ gegeben, und im Finstern eine solche Kopfbewegung ausgeführt, dass die Linie vertikal erscheint, und lässt. nun, obne seine Stellung zu ändern, die Thür öffnen, so dass die Tische, Fensterbriustungen u. s. w. sichtbar werden, so geht die Linie augenblicklich in die schiefe Lage zurück. Lässt man die Thür wieder schliessen, während man in derselben Stellung verharrt, so wird die helle Linie in 1-2 Sekunden wieder vertikal. Ich habe das sehr oft hintereinander mit constantem Erfolge wiederholt und auch von andern Personen bestätigen lassen. Noch frappanter ist der Versuch in folgender Weise: bei geöffneter Thür, so dass die Meubles im Zimmer sichtbar sind, fixirt man die schief gestellte Linie und neigt den Kopf um etwa $90^{\circ}$ : die Linie erscheint schief. Nun hält man ein gefärbtes (blaues oder rothes) Glas von dunklerer Nüance vor die Augen: sogleich beginnt der Streifen sich zu drehen und hat binnen 1-2 Sekunden eine vertikale Stellung eingenommen. Nimmt man das verdunkelnde Glas hinweg, so geht die Linie sofort zu ihrer schiefen Lage zurück. - Durch das gefärbte Glas werden nämlich die matt erleuchteten Gegenstände im Zimmer ganz unsichtbar, ebenso wie bei geschlossener Thür, während die lichtstarke helle Linie sehr deutlich bleibt. Dies ist also nur eine andere Form des Versuches, die ihrer Bequemlichkeit wegen zu empfehlen ist.

Die Drehung der hellen Linie ist unabhängig von allen äbrigen Kopf- und Augenbewegungen. Mag man 
den Kopf um die vertikale oder horizontale Axe drehen, oder die Augen eine solche Bewegung ausführen lassen; die scheinbare Lage der hellen Linie ändert sich nicht. Hat man andererseits die Grundlinie nach rechts oder links geneigt und hat die helle Linie dadurch ihre Lage scheinbar verändert, so wird durch alle möglichen übrigen Bewegungen des Kopfes oder der Augen keine Veränderung in der scheinbaren Lage der hellen Linie hervorgebracht. Die Erscheinung ist also nur abhängig von der Neigung des Kopfes nach rechts und links oder von der Neigung der Grundlinie.

Es ist ohne Einfluss, ob man bei diesen Versuchen ein Auge oder beide anwendet.

Einen Einfluss der Vorstellung oder der Aufmerksamkeit habe ich nicht bemerken können. Die veränderte Lage der Linie wird mit dem Zwange einer unmittelbaren Sinneswahrnehmung dem Beobachter aufgedrängt, und lässt sich durch innere Vorgänge ebenso wenig änderu, wie die scheinbare Grösse des Mondes bei seinem Aufgange.

Machen wir uns klar, was bei diesem Versuche in uns vorgeht, so finden wir 1) eine Drehung der Retina im Raume um die optische Axe. Bei einer solchen veränderten Lage der Retina werden wir, wenn sie obne unser Bewusstsein eintritt, alles, was sich auf dem für gewöhnlich vertikalen Meridiane abbildet, als vertikal gelegen, alles, was im horizontalen Meridiane vor sich geht, als horizontal ansehen. Hat nun die Drehung der Retina $90^{\circ}$ betragen, so werden wir unter jener Bedingung alles Vertikale für horizontal halten und umgekehrt. 2) Haben wir zu beachten, dass die Lageveränderung unserer Retina willkührlich und mit Bewusstsein geschieht, indem wir unserm Kopfe eine gewisse Neigung mittelst unserer Muskeln ertheilen. Dieser bewussten Bewegung entsprechend werden wir nun die Vorgänge auf unserer Netzhaut beurtheilen, d. h. wenn wir eine Bewegung um $90^{\circ}$ ausgeführt haben und also wissen, dass unser horizontaler Meridian vertikal liegt, so werden wir eine Linie, die ihn trifft, nicht als horizontal, sondern als vertikal ansprechen. Hierbei sind wir aber einer Täuschung ausgesetzt: wenn wir nämlich eine Neigung des Kopfes um $90^{\circ}$ beabsichtigen, so ist es 
immerhin unsicher, ob wir die beabsichtigte Rewegung wirklich in diesem Umfange ausführen; wir werden im Finstern der Gefahr ausgesetzt sein, statt der beabsichtigten Bewegung von $90^{\circ}$ in Wirklichkeit eine Bewegung von vielleicht nur $45^{\circ}$ auszuführen. Sind wir nun der Meinung, unser vertikaler Meridian liege horizontal, und er liegt in Wirklichkeit zwischen der Vertikalen und Horizontalen in der Mitte, so muss daraus eine falsche Beurtheilung der Lage von Objekten resultiren. In diesem Falle werden wir eine in Wirklichkeit um $45^{\circ}$ im Sinne unserer Drehung geneigte Linie für horizontal, eine vertikale für eine um $45^{\circ} \mathrm{im}$ Sinne unserer Drehung geneigte schiefe Linie halten. Aehnliches würde sich ereignen, wenn wir statt der beabsichtigten Drehung um $90^{\circ}$. eine wirkliche Bewegung von $135^{\circ}$ gemacht hätten, nur würde die scheinbare Drehung der hellen Linie im letzteren Fall der Bewegung unseres Kopfes entgegengesetzt sein. Eine derartige falsche Beurtheilung der Grösse unserer-Bewegung, und zwar ein Ueberschätzen der Bewegungsgrösse, würde unsere Erscheinung erklären. Zur Prüfung dieser Möglichkeit stellte ich folgende Versuche an: Ich neigte vor einem Spiegel mit geschlossenen Augen den Kopf so weit, dass ich glaubte, er sei um $90^{\circ}$ geneigt, oder die Grundlinie stehe vertikal: als ich die Augen öffnete, war dies wirklich der Fall; ich hatte demnach die Grösse der Bewegung richtig beurtheilt. - Nun erzeugte ich im finstern Zimmer ein vertikales Nachbild von einer Lichtflamme und blickte ohne Neigung des Kopfes nach der um $45^{\circ}$ geneigten hellen Linie, so dass sich das Nachbild und die helle Linie durchkreuzten. Ich neigte den Kopf um $90^{\circ}$, wie ich glaubte: die belle Linie erschien vertikal, das Nachbild schief. Ich liess die Thür, obne meine Stellung zu ändern, öffnen: sofort wurde die helle Linie schief und das Nachbild horizontal. Ich änderte den Versuch so $a b$, dass ich bei geöffneter Thür ein vertikales Nachbild erzeugte und den Kopf so lange neigte, bis es horizontal erschien: die helle Linie erschien schief, das Nachbild horizontal. Nun hielt ich ein dunkelblaues Glas vor die Augen: sogleich begannen die helle Linie und das Nachbild ihre Richtung zu ändern, so dass in wenigen Sekunden die helle Linie vertikal, das Nachbild schief 
erschien. Dies konnte ich, da ich durch viele Versuche schon ziemlich empfindlich für Nachbilder geworden bin, mit ein und demselben Nachbilde mehrere Male hintereinander ausführen: i mm er wurde die helle Linie beim Vorbalten des Glases vertikal, das Nachbild schief; bei Wegnahme des Glases die helle Linie schief, das Nachbild horizontal. Auch in diesen Versuchen hatte ich also die Grösse der mit dem Kopfe ausgefuthrten Bewegung richtig beurtheilt.

Erzeugte ich nun ein vertikales Nachbild und neigte den Kopf vor dem Spiegel um $90^{\circ}$, so erschien das Nachbild horizontal. Ich schloss die Augen: nach Verlauf einiger Sekunden stand das Nachbild, ohne dass ich eine Bewegung mit dem Kopfe gemacht hatte, schief.

Hieraus geht nun wohl hervor, dass die scheinbare Drehung der hellen Linie nicht direct auf einer falschen Schätzung der von uns ausgeführten Bewegung beruhen kann, denn wenn auch wirklich kleine Fehler dabei vorkommen, so führen wir sicherlich nicht statt einer Drehung von $90^{\circ}$ eine Drehung von $135^{\circ}$ aus, was der Fall sein müsste, wenn wir daraus unsere Erscheinung erklären wollten.

Die scheinbare Drehung der Nachbilder lässt uns aber an ein drittes Moment denken, nämlich an eine Drehung der Bulbi um die optische Axe. Es wäre denkbar, dass der Bulbus eine der des Kopfes entgegengesetzte Drehung ausführte, so dass bei einer Drehung der Grundlinie eine entgegengesetzte Drehung des vertikalen Meridians einträte. In unserem Falle müsste nun bei einer Drehung der Grundlinie um $90^{\circ}$ eine entgegengesetzte Drehung des Bulbus oder des vertikalen Meridians um $45^{\circ}$ stattfinden. Es würde der Effect alsdann derselbe sein, den wir bei der Annahme einer falschen Scbätzung unserer Kopfbewegung gefunden haben, denn auch hier würde statt der angenommenen Drehung der Retina um $90^{\circ}$ nur eine Drehung von $45^{\circ}$ stattfinden, welcher entsprechend wir die Lage der uns umgebenden objecte beurtheilen würden. Dagegen spricht aber erstens, dass bei Neigung des Kopfes vor einem Spiegel eine Drehung der Iris nicht beobachtet wird (Tourtual, Donders), zweitens, dass sich das Nachbild, wenigstens im 
Hellen, ebenso dreht, wie die Grundlinie (Donders). Drittens erzeugten wir, auf Herrn Professor Volkmann's Vorschlag, bei vertikal stehender Grundlinie ein Nachbild von der in Wirklichkeit vertikalen, scheinbar aber schiefstehenden, hellen Linie: beim Aufrichten des Kopfes (also horizontaler Grundlinie) erschien das Nachbild deutlich horizontal. Viertens ist eine derartige Drehung des Bulbus, welche im Ganzen über $90^{\circ}$ betragen müsste, schon wegen der Befestigung des Nervus opticus am Bulbus eine anatomische Unmöglichkeit. - Endlich würde aber eine compensirende, der des Kopfes entgegengesetzte Bewegung des Bulbus unsere Erscheinung nicht erklären, da vielmehr nach dem oben Erörterten eine grössere Drehung der Retina, als sie durch Bewegung des Kopfes hervorgebracht wird, eintreten müsste. Eine Drehung des Bulbus um die optische Axe findet also bei Neigung des Kopfes nach rechts und links nicht statt, und könnte unser Phänomen nicht erklären.

Wir werden zu einem anderen Gesichtspunkte gelangen, wenn wir die Art und Weise, in der die veränderte Lage der hellen Linie eintritt, genau betrachten. Die Erscheinung ist nämlich so, dass wir nicht eigentlich eine Drehung der hellen Linie wahrnehmen, wie sie uns bei einer wirklichen Drehung eines Objectes erscheint. Es macht vielmehr den Eindruck, als ob die helle Linie an 0 rt und Stelle bliebe, dagegen der umgebende Raum sich drehte, oder unsere Vorstellung von oben und Unten, Rechts und Links sich veränderte, oder sit venia verbo! sich drehte. Diesen Eindruck hat der Versuch nicht nur mir, sondern auch allen meinen Freunden gemacht, die ich darum befragte. Die eigenthïmliche Veränderung, welche in unserer Vorstellung von Oben und Unten eintritt, zeigte sich bei einer kleinen Abänderung des Versuches noch deutlicher. Liess ich nämlich an dem verschlossenen Fenster etwas mattes Licht nach oben gegen den Bogen der Mauer uber dem Fenster scheinen, stellte mich gerade vor das Fenster und neigte den Kopf nach rechts, so schien das matte Licht nicht mehr vor mir zu sein, da wo ich es bei aufrecht stehendem Kopfe sah, sondern um eine bedeutende Strecke weiter nach links zu liegen, während die Region, die vor und über mir zu sein schien, ganz dunkel war. Wenn 
ich aber einen hellen Fleck vor mir nach links hin verlege, so geht daraus wohl hervor, dass meine Vorstellung von oben und Unten eine unrichtige geworden ist. Dieser falschen Vorstellung von den Richtungen des Raumes gemäss müssen wir nun die Lage von Bildern auf unserer Retina deuten. Aendert sich demnach unsere Vorstellung in der Weise, dass wir das Oben unserer Vorstellung nach links hin und zwar nach links und oben verlegen, so werden wir auch bei einer vertikalen Linie, die wir fixiren, ihren obersten Punkt nach oben und links, und entsprechend ihren untersten Punkt nach unten und rechts hin verlegen und das Oben unserer veränderten Vorstellung wird dem wirklichen oben und Rechts entsprechen. Der Vorgang würde sich in uns also so gestalten: durch die Neigung unseres Kopfes ändert sich unsere Vorstellung von den Richtungen des Raumes (woher das kommt, werden wir sogleich untersuchen) und dieser veränderten Vorstellung gemäss beurtheilen wir die Lage uns unbekannter Objecte.

Sind wir dagegen von bekannten Objecten umgeben, so werden dieselben uns fortwährend Anhaltspunkte für die Berichtigung unserer Vorstellung bieten, und wir werden daher im hellen Zimmer, wie wir auch unsern Kopf neigen mögen, einer solchen Täuschung nicht unterliegen. Durch diese Auffassung verliert die Drehung des Nachbildes beim Schliessen der Augen das Befremdende, denn von unserer Vorstellung vom Raume ist ja auch die Lage eines jeden unserer Retina gehörenden Bildes abhängig, mag dasselbe von einem wirklichen Objecte abhängen oder subjectiver Natur sein; die Vorstellung ist gewissermaassen die höhere Instanz für die Retina, ändert sich die Vorstellung von den Richtungen des Raumes, so muss sich damit die Auslegung der Retinaeindrücke ändern. So ändert sich ja auch die Grösse eines Nachbildes, je nachdem wir es in grössere oder geringere Entfernung projiciren, so ändert sich auch die Grösse eines Bildes von einem wirklichen Objecte, wenn wir im Stande sind, unserer Vorstellung freies Spiel über die Entfernung desselben zu gestatten. Ist dagegen unsere Vorstellung gebunden, so sind wir gezwungen, unsere Retinaeindrucke in einer bestimmten Weise ihr gemäss auszulegen, haben wir z. B. 
von dem Himmelsgewölbe die Vorstellung, dass es in der Form eines Uhrglases über uns ausgebreitet sei, so müssen wir das gleich grosse Bild des Mondes auf unserer Retina für verschieden gross halten, wenn uns die Vorstellung, die wir von dem Gewölbe haben, zwingt, ihn in grössere oder geringere Entfernung zu projiciren.

Wir müssen nun untersuchen, wie wir dazu kommen, unsere Vorstellung von Oben und Unten, von Rechts und Links umzugestalten, respective, was unszwingt, sie umzugestalten? Wir kommen auf zwei Wegen zu der siunlichen Vorstellung von den Richtungen des Raumes, durch die Bewegungen unserer Retina und durch die Bewegungen unseres übrigen Körpers. Die Bewegungen unserer Retina sind aber zum Theil von denen unseres Körpers abhängig, zum Beispiele bei den Neigungen unseres Kopfes. Soll keine Disharmonie zwischen beiderlei Vorstellungen eintreten, so muss ein fortwährender Rapport zwischen ihnen stattfinden. Dieser Rapport wird vermittelt durch den Tastsinn. Er giebt uns Nachricht von den Bewegungen unseres Körpers, und aus den erfolgten oder nicht erfolgten Bewegungen schliessen wir auf die Lage unseres Körpers, und auf die Lage der ausser uns befindlichen Objecte, welche den Tastsinn oder den Gesichtssinn afficiren. Bewegungen unseres Körpers, die uns nicht gemeldet werden, müssen daher eine Desorientirung unserer Vorstellungen zur Folge haben, wofür es bekanntlich viele Beispiele und Erfahrungen giebt. - Indess nicht nur Bewegungen, die uns nicht gemeldet sind, werden uns desorientiren, sondern auch Bewegungen, die uns zwar gemeldet worden sind, die wir aber vergessen haben. Ein solches Vergessen kann sowohl seitens des Verstandes, als seitens der Sinnlichkeit stattfinden, und hierauf glaube ich meine Erklärung des uns beschäftigenden Phänomens begründen zu müssen. Wir haben gesehen, dass die Bewegungen, die wir mit dem Kopfe nach rechts und links machen, uns richtig gemeldet werden, es folgt daraus aber nicht, dass wir uns der ausgeführten Bewegung daúernd bewusst bleiben, wir werden vielmehr geneigt sein, eine Situation, in die wir durch Bewegungen gekommen sind, zu vergessen, wenn es uns an Erinnerungsobjecten fehlt, von denen 
wir fortdauernd orientirt erhalten werden. Denken wir an das alte Aristotelische Experiment der zwischen den verschränkten Fin- * gern doppelt erscheinenden Kugel. Wir wissen seitens des Verstandes, dass eine Verschränkung unserer Finger stattgefunden hat, und dass sie nicht aufgehoben worden ist. Unser Tastsinn erinnert sich, um es kurz auszudrücken, in den ersten Augenblicken auch noch daran, denn die ersten Bewegungen, die wir mit der Kugel machen, erzeugen noch nicht die Vorstellung von zwei Kugeln; diese tritt erst nach wiederholten Bewegungen ein. Es wird also mit der Zeit die Erinnerung in unserem Tastsinne ausgelöscht, dass die Finger verschränkt sind und nur dadurch wird die Täuschung möglich. Aehnlich ist es beim Schielen. Wir wissen auch hier, dass wir Bewegungen ausführen, aber diese Bewegungen werden uns in viel geringerem Grade bewusst, als die Bewegungen bei dem Versuche mit der Kugel, namentlich bleibt unsep Tastsinn fast ganz ununterrichtet darüber, und daraus wird es begreiflich, wạum die Doppelbilder beim Schielen viel zwingender auftreten, als in jenem Versuche die Doppelkugeln. Offenbar ist die Verschränkung der Finger ein viel bedeutenderer Eindruck aut den Tastsinn, als die kleine Verschiebung und Spannung der Conjunctiva beim Schielen.

Wenden wir diese Betrachtungen auf unser Experiment an und halten dabei fest: 1) dass wir bei der Neigung unseres Kopfes eine wirkliche Drehung der Netzhaut im Raume ausgeführt haben, so dass der vertikale Meridian horizontal liegt, 2) dass uns diese Bewegung zum Bewusstsein gekommen ist. - Wäre Letzteres nicht der Fall, so würden wir alles Vertikale für horizontal halten; dass es nicht geschieht, kommt daher, dass der Rapport zwischen den Körperbewegungen und der Augenbewegung hergestellt worden ist. Aber wir dürfèn nicht vergessen, dass dieser Rapport nicht aufhören darf, und er wird auch unterbalten erstens durch das sogenannte Muskelgefühl (den Tastsinn), zweitens durch die im Hellen das Gesichtsfeld erfílilenden bekannten Objecte. Im finstern Zimmer fällt dieses zweite Moment weg, es bleibt uns zu unserer Orientirung nur das Muskelgefühl oder die Eindrücke auf den Tastsinn. Je länger wir in dieser Lage bleiben, um so mehr wird der Ein- 
fluss derselben auf unsere Vorstellung schwinden, es wird ein Wettstreit zwischen der um $90^{\circ}$ gedrehten Retina und dem Muskelgefühl entstehen, wodurch unsere Vorstellung von dem Oben und Unten in dem finstern Raume verändert werden muss. Die Retina wird die Tendenz haben, dahin die vertikale Richtung des Raumes zu versetzen, wo ihr sonst vertikaler Meridian liegt, also in die horizontale Richtung; das Muskelgefühl oder der Tastsinn werden ihr entgegenwirken, und die wirklich vertikale Richtung in ihre Rechte einzusetzen suchen. Kurz: in dem Grade, wie wir aufhören, uns der Lage unseres Kopfes von Seiten der Sinnlichkeit zu erinnern, wird die vorhandene Drehung der Retina unsere Vorstellung verändern. Wir werden um so mehr geneigt sein, unser Muskelgefübl unbeachtet zu lassen, weil wir im gewöhnlichen Leben uns bei Neigungen des Kopfes viel mehr nach den uns bekannten Objecten über den Raum orientiren, als nach dem Muskelgefübl. Immerhin wird aber das Muskelgefübl einigen Einfluss auf unsere Vorstellung behalten, und wir werden nicht nach der Lage unserer Retina allein urtheilen, sondern wir werden uns eine mittlere, aus beiden Momenten resultirende, Vorstellung von dem Oben und Unten bilden, und also bei rechts geneigtem Kopfe das Oben nach rechts und oben, das Unten nach links und unten verlegen. Dann wird aber, wie gesagt, eine wirklich vertikale Linie von links oben nach rechts unten geneigt erscheinen müssen.

So wie wir nun in das finstere Zimmer Licht einlassen, so wird sich zu unserem Muskelgefühl die Orientirung aus bekannten Objecten gesellen, und unsere Vorstellung wird wieder berichtigt werden.

Für diese Deutung kann ich nun noch Folgendes anführen:

1) Die Abweichung oder scheinbare Drehung der hellen Linie nimmt bei längerer Dauer der Neigung des Kopfes immer mehr zu. Tragen wir Sorge,' dașs unser Gefühl von der Veränderung unserer Lage verIoren gebt, so haben wir zu erwarten, dass uns bei horizontaler Lage unseres vertikalen Meridians eine vertikale Linie horizontal erscheint. In der That; legte ich mich lang ausgestreckt auf den Fussboden des verfinsterten Zimmers, liess den 
Kopf mit vertikaler Grundlinie auf einem weichen Kissen ruhen, und hielt ein dunkles Glas vor die Augen, so dass ausser der hellen Linie nichts $z u$ sehen war: so wurde im Verlaufe einiger Minuten die helle Linie immer stärker gegen ihre wirkliche Lage geneigt, so dass sie nur etwa $10^{\circ}$ von der horizontalen Richtung abzuweichen schien; ganz horizontal wurde sie nicht. Sie ging indess hin und wieder von der horizontalen Richtung mehr zur vertikalen Richtung zuruck, woran, wie ich glaube, Bewegungen des Körpers oder Kopfes die Ursache waren, indem sie die Lage des Körpers in Erinnerung brachten. 2) die scheinbare Drehung der bellen Linie ist bei mirnicht jedesmal gleich gross; während ich meist die Abweichung auf etwa $45^{\circ}$ schätzen musste, schien sie 'mir andere Male viel weniger zu betragen, etwa nur $25^{\circ}-30^{\circ}$. Die Bedingungen dieser Verschiedenheit habe ich noch nicht ermitteln können. 3) sie ist individuell verschieden. Meinem Freunde und Collegen Leopold Auerbach schien die Drekung der hellen Linie bei senkrechter Grundlinie nur etwa $15^{\circ} \mathrm{zu}$ betragen, nahm aber bei längerer Dawer der Neigung des Kopfes allmälig beträchtlich zu, sowie auch, wenn derselbe den Kopf stärker neigte. Auerbach bemerkte auch während der Neigung des Kopfes Oscillationen in der Abweichung der Linie, so dass sie sich bald mehr, bald weniger von der Vertikalen zu entfernen schien. Ich selbst habe dergleichen 0scillationen nicht mit Sicherheit bemerken können, ausser in dem Falle, wo ich lang ausgestreckt auf dem Fussboden lag.

Nehmen wir die Deutung, dass sich bei Neigung des Kopfes nach rechts und links unsere Vorstellung von den Richtungen des Raumes ändert, und diess auf einer Disharmonie der durch die Netzhaut und der durch Muskelgefühl oder Tastsinn vermittelten Vorstellungen beruht, an, so erklären sich daraus folgende Erscheinungen des Versuches:

1) die der Neigung unserer Grundlinie entgegengesetzte Richtung der Drehung der hellen Linie.

2) dass die scheinbare Drehung der hellen Linie nur etwa 
halb so viel beträgt, als die Neigung des Kopfes oder die Drehung des vertikalen Meridians.

3) dass die Grösse đer scheinbaren Drehung individuell verschieden, und auch bei demselben Individuum nicht immer gleich gross ist.

4) dass die Grösse der scheinbaren Drebung mit der Dauer der Neigung zunimmt.

5) dass auch die Nachbilder einer solchen Drehung unterworfen sind.

6) dass die Erscheinung nur im Finstern erfolgt, wenn wir nicht von bekannten Objecten umgeben sind, und sogleich schwindet, wenn wir dergleichen wahrnehmen.

7) dass die Drehung der hellen Linie unabhängig ist von den übrigen Kopf- und Augenstellungen.

Indem ich mir eine weitere Untersuchung des Phänomens vorbehalte, an die ich Untersuchungen über die Stellung des vertikalen Meridians bei Neigung des Kopfes nach rechts und links anschliessen werde, bemerke ich noch, dass die Herren Dr. Auerbach, Professor Heidenhain, Dr. Neumann und Professor Volkmann die Güte gehabt haben, mir die Erscheinung zu bestätigen.

Breslau, den 24. October 1860. 